Review

\title{
Ge-Photodetectors for Si-Based Optoelectronic Integration
}

\section{Jian Wang and Sungjoo Lee *}

Department of Electrical and Computer Engineering, National University of Singapore, 4 Engineering Drive 3, 117576 Singapore, Singapore; E-Mail: wangjian@nus.edu.sg

* Author to whom correspondence should be addressed; E-Mail: elelsj@nus.edu.sg; Tel.: +65-6516-6140; Fax: +65-6779-1103.

Received: 5 November 2010; in revised form: 29 November 2010 / Accepted: 10 January 2011 / Published: 12 January 2011

\begin{abstract}
High speed photodetectors are a key building block, which allow a large wavelength range of detection from $850 \mathrm{~nm}$ to telecommunication standards at optical fiber band passes of 1.3-1.55 $\mu \mathrm{m}$. Such devices are key components in several applications such as local area networks, board to board, chip to chip and intrachip interconnects. Recent technological achievements in growth of high quality $\mathrm{SiGe} / \mathrm{Ge}$ films on $\mathrm{Si}$ wafers have opened up the possibility of low cost Ge-based photodetectors for near infrared communication bands and high resolution spectral imaging with high quantum efficiencies. In this review article, the recent progress in the development and integration of Ge-photodetectors on Si-based photonics will be comprehensively reviewed, along with remaining technological issues to be overcome and future research trends.
\end{abstract}

Keywords: germanium; photodetector; Si photonics

\section{Introduction}

In the past decade, Si photonics has become one of the hottest research domains in the World since it holds great promise for maintaining the performance roadmap known as Moore's Law. As short-distance data exchange rates approach $10 \mathrm{~Gb} / \mathrm{s}$, metal interconnection is facing a number of inevitable issues such as slow resistance-capacitance limit speed and large heat dissipation. Under these circumstances, it is well known that for data communication beyond $10 \mathrm{~Gb} / \mathrm{s}$, optical signal delivery is more advantageous compared to today's copper interconnections. As a result, combining sophisticated process techniques, low cost and mass production, Si based Electro-Photonic Integrated 
Circuits (EPIC) emerge as one of the most promising solutions for next generation interconnection techniques. In fact, long-haul combinations have been based on fiber optics techniques for the last 30 years. The wavelength used for the majority of long-distance data transitions is in the 1.3-1.55 $\mu \mathrm{m}$ range, corresponding to the minimum loss window of silica optical fiber. If the same wavelength can be utilized in the future short-distance data transfers including inter-chip, chip-to-chip and Fiber-To-The-Home (FTTH) communications, all end users will be able to connect directly to the external servers without the need for wavelength conversion, making global communication much easier and cheaper. As a result, Si EPIC working in 1.3-1.55 $\mu \mathrm{m}$ wavelength has become aggressively pursued by researchers worldwide.

To date, enormous efforts have been invested in Si photonics techniques and critical breakthroughs and milestones have been achieved. Various passive components [1], active devises like lasers [2], and high speed modulators [3] have been reported. Being the device that ends the optical path, photodetectors, which convert light back into electrical signals, are vital component for Si photonic integrated circuits. In fact, the trigger of the past decade's Si photonics upsurge was the first successful demonstration of the high-efficiency Germanium photodetector [4]. Although Si photodetectors have been widely used in optical receivers in the wavelength range around $850 \mathrm{~nm}$, its relatively large bandgap of $1.12 \mathrm{eV}$ corresponding to an absorption cutoff wavelength of $\sim 1.1 \mu \mathrm{m}$ hinders $\mathrm{Si}$ photodetectors' application in the longer wavelength range of 1.3 and $1.55 \mu \mathrm{m}$. For a more seamless integration with current long-haul communication technology, a material with strong absorption coefficient in the $1.30-1.55 \mu \mathrm{m}$ is very desirable.

Among the available choices, III-V compound semiconductors possess the advantage of high absorption efficiency, high carrier drift velocity and mature design and fabrication technology for optical devices. Therefore, integration of high performance III-V photodetectors onto the Si platform by flip-chip bonding or direct heteroepitaxy has been widely reported. However, the introduction of III-V materials into Si process is at the expense of high cost, increased complexity and potential introduction of doping contaminants into the Si CMOS devices since III-V materials also act as dopants for group IV materials.

Germanium, a group IV material the same as $\mathrm{Si}$, avoids the cross contamination issue. Though Ge is also an indirect bandgap $\left(\mathrm{E}_{\mathrm{g}}=0.66 \mathrm{eV}\right)$ material like $\mathrm{Si}$, its direct bandgap of $0.8 \mathrm{eV}$ is only $140 \mathrm{meV}$ above the dominant indirect bandgap. As a result, Ge offers much higher optical absorption in 1.3-1.55 $\mu \mathrm{m}$ wavelength range, thus making Ge-based photodetectors promising candidates for $\mathrm{Si}$ photonics integration. However, the $4 \%$ lattice mismatch between $\mathrm{Ge}$ and $\mathrm{Si}$ places challenging obstacle towards monolithic integration of high-quality low dislocation density Ge devices through Ge on Si heteroepitaxy. Nevertheless, to date, device-grade single-crystalline Ge films have been demonstrated by many groups with practical high performance Ge photodetectors.

In this review paper, we first introduce in Section 2 the various Ge growth techniques. Different photodetector electrical structures and light coupling schemes are briefly described in Sections 3 and 4, respectively. In Section 5, the historical research trends along with the performances of $\mathrm{Ge}$ photodetectors reported by various research groups are summarized, along with the remaining technical issues and future research directions. Conclusions are presented in Section 6. 


\section{Ge Growth Techniques}

Tracing back in history, the first Ge on Si detector was reported in 1984 by Luryi et al. [5]. The demonstrated detector showed $41 \%$ quantum efficiency at a wavelength of $1.45 \mu \mathrm{m}$, where an MBE-grown 1,800 $\mathrm{A}+\mathrm{Ge}_{\mathrm{x}} \mathrm{Si}_{1-\mathrm{x}}$ alloy (graded in ten steps from $\mathrm{x}=0$ to $\mathrm{x}=1$ ) acted as a buffer layer for the heteroepitaxy of Ge on Si. Since then, various techniques with their own pros and cons have been pursued for the growth of Ge films on Si surfaces. The main quality criterion of the Ge layer can be categorized as: procedure complexity, material cost, growth temperature, and the resulting Ge layer's dislocation density and strain.

\subsection{Poly Ge Films}

For ease of integration of near-infrared detectors with standard Silicon process lines for signal acquisition, amplification and processing, low temperature growth of Ge layers is much desired. In 2000, a Ge deposition approach based on the thermal evaporation with process temperatures as low as $\sim 300{ }^{\circ} \mathrm{C}$ was first proposed in the pioneering work conducted by Masini et al. [6]. It was found that polycrystalline Ge deposition can be possible at substrate temperatures as low as $300{ }^{\circ} \mathrm{C}$, as confirmed by the Raman spectra results (Figure 1). This method allows simple and low cost integration with Si processes. Monolithic integration of an array of eight polycrystalline Ge pixels with CMOS readout electronics was demonstrated based on this method [7], shortly after which Colace et al. [8] reported the realization of a digital camera, further confirming the process compatibility of the low-temperature approach.

Figure 1. (a) Raman spectra of the Ge on Si samples grown at different temperatures by thermal evaporation method. From [6]. (b) Photograph of one pixel of the digital camera (top) and a sketch of its cross section. From [8].

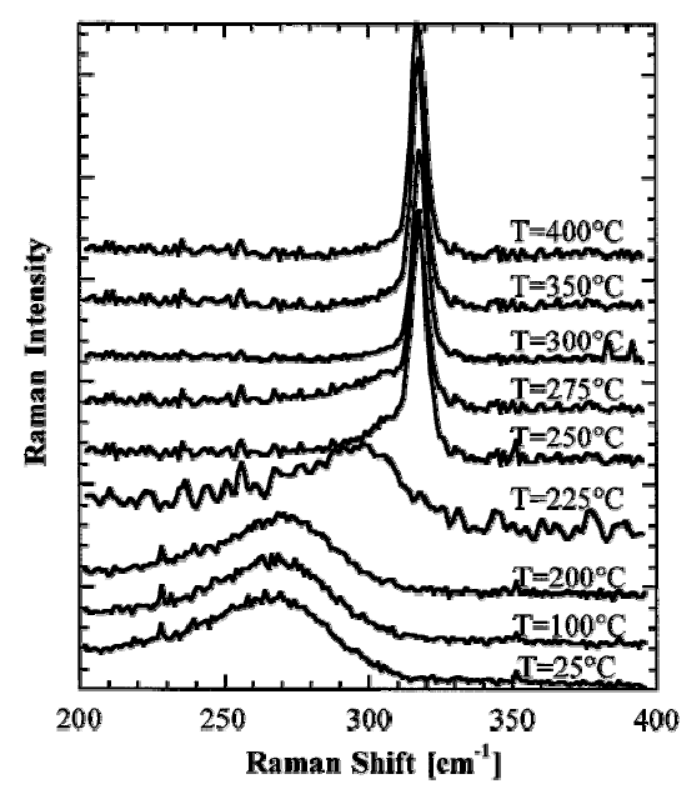

(a)

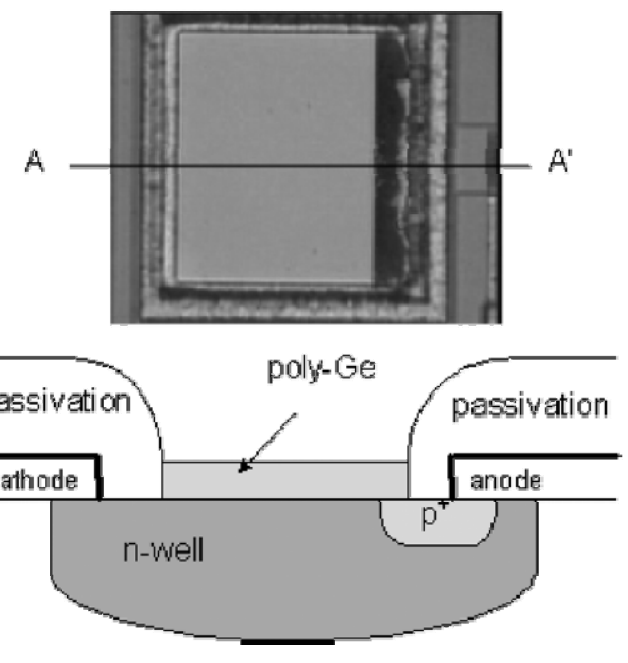

(b) 
Moreover, although the low temperature deposition introduces a relatively high density of defects and dislocations into the poly-Ge layer and worsens the electrical properties compared to crystalline Ge films, it was shown recently that by a careful design, acceptable performance of the polycrystalline Ge photodetector for Si photonics integration can be obtained, with responsivities between $0.1 \mathrm{~A} / \mathrm{W}$ and $0.3 \mathrm{~A} / \mathrm{W}[9]$.

\subsection{Crystalline Ge Growth with Graded SiGe Buffer Layers}

In the early stage of crystalline Ge film epitaxy on Si wafers, a compositionally graded SiGe region was commonly adopted as buffer layer. This approach was first adopted in the SiGe/Si system by Luryi et al. [5] and later improved by Fitzgerald et al. in 1990 [10]. Multiple buffer layers with increasing Ge content were adopted to relax the high strain between $\mathrm{Ge}$ and $\mathrm{Si}$, which minimizes dislocation nucleation and reduces the threading dislocations. The final strain-relaxed $\mathrm{Si}_{1-\mathrm{x}} \mathrm{Ge}_{\mathrm{x}}$ layers grown on these graded layers showed low density of threading-dislocations, $4 \times 10^{5} \mathrm{~cm}^{-2}$ for $\mathrm{x}=0.23$ and $3 \times 10^{6} \mathrm{~cm}^{-2}$ for $\mathrm{x}=0.50$.

However, the graded SiGe buffer method usually requires a thick $10 \mu \mathrm{m}$ buffer for pure Ge epitaxy on Si, while in modern Si photonics technology, Ge photodetectors are favorably fabricated in close adjacency with Si optical waveguide facilitating evanescent or butt-coupling of the optical power. As a result, a new technique with thin buffer layers is still needed.

\subsection{Two Step LT/HT Ge Growth}

The origin of the two-step LT/HT (low temperature/high temperature) growth technique can be traced back to 1986 for GaAs growth on Si by Fan et al. [11]. Its application in the epitaxially grown Ge on Si was first proposed and utilized by Colace et al. [12] in a ultra high vacuum chemical vapor deposition (UHVCVD) growth reactor in 1998, since when it has attracted wide interest for Ge epitaxial growth. In the two-step Ge growth procedure, first, after thorough cleaning, the substrate is maintained at low temperature $\left(\sim 300-400{ }^{\circ} \mathrm{C}\right)$, and a thin layer of Ge buffer layer $(\sim 50-100 \mathrm{~nm})$ is grown to prevent strain release through undesirable island growth. Second, the substrate temperature is elevated to $\sim 550-700{ }^{\circ} \mathrm{C}$ and a thick Ge layer with reduced threading dislocation density is grown on top of the low-temperature thin Ge buffer. It should be noted that the two-step Ge method can be adopted not only in UHVCVD systems, but also in growth tools such as reduced-pressure CVD (RPCVD) [13] and molecule beam epitaxy (MBE) [14].

The Ge layers growth by two-step Ge epitaxy typically suffers from a high threading dislocation density (TDD) in the order of $10^{8}-10^{9} \mathrm{~cm}^{-2}$. Therefore, high temperature annealling is employed by many groups to reduce the TDD to an acceptable level. For example, the research of Luan et al. indicates that the TDD in two-step Ge layer can be significantly reduced by cyclic thermal annealing. The optimized annealing condition $\left(900{ }^{\circ} \mathrm{C} / 10 \mathrm{~min}, 780{ }^{\circ} \mathrm{C} / 10 \mathrm{~min}\right.$, cycle number: 10$)$ can reduce the threading dislocation density to $\sim 2 \times 10^{7} \mathrm{~cm}^{-2}[15,16]$. Ge photodetectors based on this process were successfully demonstrated to have improved performance $[15,17]$. However, the annealing process increases the thermal budget undesirable for photodetectors' integration with Si MOSFET. Therefore, a number of experiments have been reported to demonstrate high Ge detector performance which are 
based on low-temperature anneal or even no additional thermal anneal $[18,19]$. In Table 1 , some of the currently active groups' Ge growth methods are summarized.

Table 1. Summary of recent Ge epitaxy method from selected groups.

\begin{tabular}{|c|c|c|c|c|c|c|c|c|c|c|}
\hline & Group & Year & Ref. & Tool & $\begin{array}{c}\text { Low Temp. } \\
\text { buffer }\end{array}$ & $\begin{array}{c}\text { High Temp. } \\
\text { Ge } \\
\end{array}$ & Anneal & Aneal condition & $\begin{array}{l}\text { RMS } \\
(\mathrm{nm}) \\
\end{array}$ & $\begin{array}{r}\text { TDD } \\
\left(\mathrm{cm}^{-2}\right) \\
\end{array}$ \\
\hline \multirow{11}{*}{ 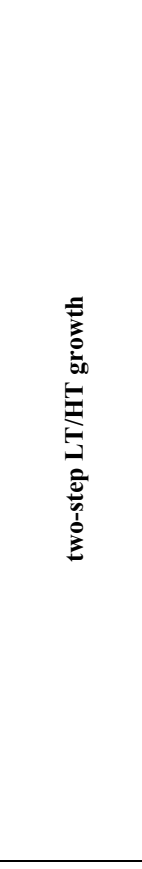 } & IEF & 2004 & [13] & RPCVD & $\begin{array}{l}400^{\circ} \mathrm{C} \\
25 \mathrm{~nm} \mathrm{Ge}\end{array}$ & $\begin{array}{l}<750{ }^{\circ} \mathrm{C} \\
730 \mathrm{~nm}\end{array}$ & yes & $\begin{array}{l}750 / 875^{\circ} \mathrm{C}, \\
10 \text { cycles }\end{array}$ & 2.2 & $<2 \times 10^{8}$ \\
\hline & IEF & 2009 & [20] & RPCVD & $\begin{array}{l}400^{\circ} \mathrm{C} \\
40 \mathrm{~nm} \mathrm{Ge}\end{array}$ & $\begin{array}{l}730^{\circ} \mathrm{C} \\
300 \mathrm{~nm}\end{array}$ & yes & not specified & - & - \\
\hline & Intel & 2006 & [21] & RPCVD & $\begin{array}{l}400^{\circ} \mathrm{C} \\
100 \mathrm{~nm} \mathrm{Ge}\end{array}$ & $\begin{array}{l}670{ }^{\circ} \mathrm{C} \\
1.2 \mu \mathrm{m}\end{array}$ & yes & $900^{\circ} \mathrm{C}, 15 \mathrm{~min}$ & - & $\sim 1 \times 10^{7}$ \\
\hline & IBM & 2004 & [22] & UHVCVD & $\begin{array}{l}350^{\circ} \mathrm{C} \\
50 \mathrm{~nm} \mathrm{Ge}\end{array}$ & $\begin{array}{l}600^{\circ} \mathrm{C} \\
400 \mathrm{~nm} \\
\end{array}$ & yes & $\begin{array}{l}780 / 900{ }^{\circ} \mathrm{C}, \\
10 \text { cycles }\end{array}$ & - & $\sim 1 \times 10^{8}$ \\
\hline & Univ. stuttgart & 2005 & [14] & MBE & $\begin{array}{l}\text { thin LT } \\
\text { buffer }\end{array}$ & $\begin{array}{l}550^{\circ} \mathrm{C} \\
1 \mu \mathrm{m} \\
\end{array}$ & no & - & - & - \\
\hline & MIT & 1999 & [16] & UHVCVD & $\begin{array}{l}350^{\circ} \mathrm{C} \\
30 \mathrm{~nm} \mathrm{Ge}\end{array}$ & $\begin{array}{l}600{ }^{\circ} \mathrm{C} \\
1 \mu \mathrm{m}\end{array}$ & yes & $\begin{array}{l}780 / 900{ }^{\circ} \mathrm{C}, \\
10 \text { cycles }\end{array}$ & - & $\sim 2 \times 10^{7}$ \\
\hline & MIT & 2007 & [23] & UHVCVD & $\begin{array}{l}360^{\circ} \mathrm{C} \\
60 \mathrm{~nm} \mathrm{Ge}\end{array}$ & $\begin{array}{l}730^{\circ} \mathrm{C} \\
1.1 \mu \mathrm{m} \\
\end{array}$ & yes & $\begin{array}{l}650 / 850^{\circ} \mathrm{C}, \\
\text { cyclic }\end{array}$ & - & - \\
\hline & Luxtera & 2007 & [24] & RPCVD & no buffer & $350^{\circ} \mathrm{C} 200 \mathrm{~nm}$ & no & - & - & - \\
\hline & Kotura & 2010 & [19] & CVD & $\begin{array}{l}400^{\circ} \mathrm{C} \\
100 \mathrm{~nm} \mathrm{Ge}\end{array}$ & $\begin{array}{l}670{ }^{\circ} \mathrm{C} \\
1.1 \mu \mathrm{m} \\
\end{array}$ & yes & not specified & - & - \\
\hline & ETRI & 2009 & [25] & RPCVD & $\begin{array}{l}400^{\circ} \mathrm{C} \\
100 \mathrm{~nm} \mathrm{Ge}\end{array}$ & $\begin{array}{l}650^{\circ} \mathrm{C} \\
1.2 / 1.7 \mu \mathrm{m}\end{array}$ & no & - & 1.3 & - \\
\hline & Univ. Roma Tre & 2006 & [18] & UHVCVD & $\begin{array}{l}350^{\circ} \mathrm{C} \\
\text { thin } \mathrm{Ge}\end{array}$ & $\begin{array}{l}600^{\circ} \mathrm{C} \\
1 \mu \mathrm{m}\end{array}$ & no & - & - & - \\
\hline \multirow{3}{*}{ 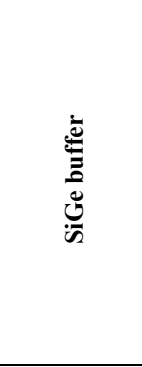 } & Unvi. Texas & 2004 & [26] & UHVCVD & $1 \mu \mathrm{m} \mathrm{SiGe}$ & $400^{\circ} \mathrm{C} 2.5 \mu \mathrm{m}$ & yes & $750^{\circ} \mathrm{C}, 15 \mathrm{~min}$ & - & - \\
\hline & Canon ANELVA & 2006 & [27] & UHVCVD & $\begin{array}{l}450-520{ }^{\circ} \mathrm{C} \\
13 \mathrm{~nm} \mathrm{SiGe} \\
370^{\circ} \mathrm{C} \\
30 \mathrm{~nm} \mathrm{Ge}\end{array}$ & $\begin{array}{l}550-600^{\circ} \mathrm{C} \\
1 \mu \mathrm{m}\end{array}$ & yes & $800^{\circ} \mathrm{C}, 15 \mathrm{~min}$ & 0.44 & - \\
\hline & IME & 2007 & {$[28]$} & UHVCVD & $\begin{array}{l}350-400{ }^{\circ} \mathrm{C} \\
30 \mathrm{~nm} \mathrm{SiGe} \\
350-400{ }^{\circ} \mathrm{C} \\
30 \mathrm{~nm} \mathrm{Ge}\end{array}$ & $\begin{array}{l}550-600^{\circ} \mathrm{C} \\
100 \mathrm{~nm}\end{array}$ & no & - & 1.4 & $\sim 1 \times 10^{7}$ \\
\hline $\mathrm{H}_{2}$ anneal & Stanford & 2008 & [29] & RPCVD & $\begin{array}{l}350^{\circ} \mathrm{C} \\
200 \mathrm{~nm} \mathrm{Ge}\end{array}$ & $\begin{array}{l}600{ }^{\circ} \mathrm{C} \\
400 \mathrm{~nm} \\
\end{array}$ & yes & $\begin{array}{l}800^{\circ} \mathrm{C}, 30 \mathrm{~min}, \\
\text { in } \mathrm{H}_{2}\end{array}$ & $\sim 1$ & $0.8-1 \times 10^{7}$ \\
\hline LEPECVD & Como & 2009 & [30] & LEPECVD & no buffer & $\begin{array}{l}500-600^{\circ} \mathrm{C} \\
1 \mu \mathrm{m}\end{array}$ & yes & $\begin{array}{l}600 / 780^{\circ} \mathrm{C}, \\
3 \text { cycles }\end{array}$ & - & $2 \times 10^{7}$ \\
\hline
\end{tabular}

\subsection{Other Ge Growth Methods}

Many attempts to modify the two-step Ge growth procedure have been reported. An UHV-CVD growth of high quality Ge on Si substrate using modified two-step Ge growth method combining with intermediate thin SiGe buffer layers was proposed first by Huang et al. in 2004 [26].The buffer region consisted of $0.6-\mu \mathrm{m}$-thick $\mathrm{Si}_{0.45} \mathrm{Ge}_{0.55}$ and 0.4 - $\mu \mathrm{m}$-thick $\mathrm{Si}_{0.35} \mathrm{Ge}_{0.65}$ layers. In-situ annealling for $15 \mathrm{~min}$ at $750{ }^{\circ} \mathrm{C}$ was carried out to further reduce the dislocation density. The thickness of the SiGe buffer was further reduced by Nakatsuru et al. [27] by employing a $13 \mathrm{~nm}$-thick $\mathrm{Si}_{0.5} \mathrm{Ge}_{0.5}$ buffer layer grown at $450-520{ }^{\circ} \mathrm{C}$. After post-deposition annealling of $800{ }^{\circ} \mathrm{C} / 15 \mathrm{~min}$, the Ge layer shows a low roughness of $0.44 \mathrm{~nm}$. Loh et al. [28] also reported an epi-Ge layer based on the SiGe buffer method, where the SiGe buffer is grown at low temperature of $350-400{ }^{\circ} \mathrm{C}$ with the thickness of around $30 \mathrm{~nm}$ (Figures 2(a,b)). 
Figure 2. (a) HR-TEM image of epitaxial Ge layer using two-step Ge growth method combining with an intermediate SiGe buffer layer. (b) Zoom-in image of the heterostructure epitaxial layers of $\mathrm{Si} / \mathrm{Si}_{0.75} \mathrm{Ge}_{0.25} / \mathrm{Ge}$. From [31].

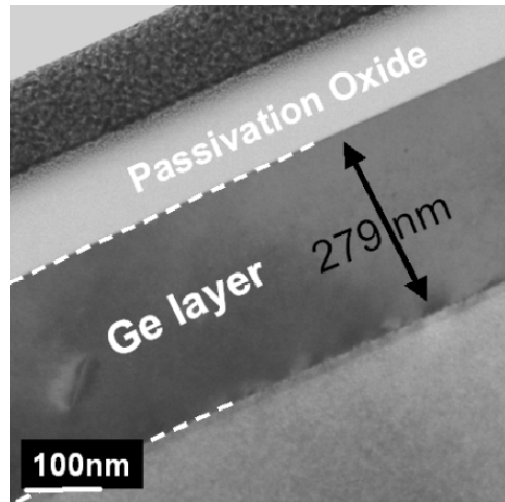

(a)

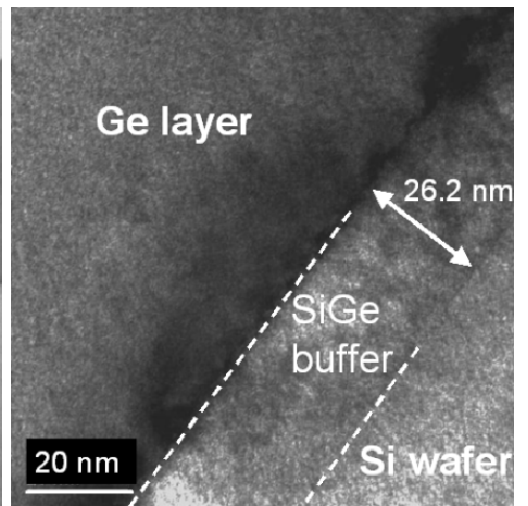

(b)

Another way to improve Ge film quality is $\mathrm{H}_{2}$ annealing, which was reported by Choi et al. [29]. The demonstrated $800{ }^{\circ} \mathrm{C} / 30 \mathrm{~min}$ anneal in $\mathrm{H}_{2}$ ambient is able to effectively improve the Ge film quality in terms of surface roughness and TDD. It is proposed that the increased atom mobility caused by hydrogen/Ge bonding is the main mechanism for the improved film surface planarity and defect density.

Recently, a new Ge epitaxy procedure based on low-energy plasma-enhanced chemical vapor deposition (LEPECVD) was demonstrated [30]. Thanks to the high deposition rates and high concentration of atomic $\mathrm{H}$ present in the chamber, Ge films with smooth surfaces and TDD $\sim 2 \times 10^{7}$ are achieved under low thermal budget. Moreover, the fabricated diode shows much lower dark current compared to the devices from UHVCVD method with comparable dislocation density. This is attributed to the improved passivation resulting from the dense plasma in the LEPECVD which is known to be efficient in generating atomic hydrogen radicals.

\section{Photodetector Electrical Structures}

Until now, a number of Ge based photodetectors with different structures are reported. Brief descriptions of typical photodetector structures will be given here for better understanding.

\subsection{PIN Detectors}

PN junctions are one of the most commonly used configurations for semiconductor photodetectors. The PIN diode where "I" stands for intrinsic, includes an intrinsic region in between the P and N regions. Due to the built-in potential or external reverse bias, the intrinsic region is depleted and has high resistivity, so that the voltage drop takes place mainly in this region, giving rise to high electric fields for effective collection of photo-generated electron-hole pairs (EHP). In this configuration, the thickness of the intrinsic region is always many times larger than the highly-doped regions so that most of the EHP's are generated within the intrinsic region where the strong electric field helps to sweep the EHP to the adjacent $\mathrm{p}+\mathrm{n}+$ region faster than diffusion. Another advantage of the PIN structure is that the depletion-region thickness (the intrinsic layer) can be tailored to optimize both the quantum 
efficiency and response bandwidth. In Ge PIN photodetectors, while the photoabsorption intrinsic layer is usually Ge for effective absorption around $1.55 \mu \mathrm{m}$, the $\mathrm{p}+$ and $\mathrm{n}+$ region can be formed either by implantation [32] or in-situ doping to form $\mathrm{p}+$ and $\mathrm{n}+$ regions for the PIN structure [14]. Another way is to use $\mathrm{p}+/ \mathrm{n}+$ single crystalline Si substrates or deposited polycrystalline Si heterojunctions [33].

\subsection{Metal-Semiconductor-Metal (MSM) Detectors}

PIN photodiodes produce a voltage drop across the diode terminals in response to an external optical input. Such devices are categorized as photovoltaic devices. On the other hand, MSM photodetectors are photoconductive devices whose conductivity is altered when an optical illumination is imposed. Therefore, MSM photodetectors are only functional under non-zero external bias. MSM photodetectors possess the advantage of low capacitance and relative ease of fabrication. The intrinsically low capacitance resulting from its configuration has always been utilized to fabricate high-speed large area detectors. One issue in early Ge MSM photodetectors was their high dark current density, which gives rise to high stand-by power consumption, thus making Ge MSM photodetectors unfavorable and impractical. Due to the narrow bandgap and strong Fermi-level pinning of the metal/Ge interface at valence band, hole injection over Schottky Barrier Height (SBH) is the major component of the dark current in Ge MSM detectors. Regarding this issue, application of dopant segregation (DS) to Ge MSM photodetectors for dark current suppression was experimentally demonstrated by Zang et al. [34-36]. Metal-Ge Schottky barrier height modification by an intermediate layer of large bandgap material such as amorphous $\mathrm{Ge}$ and $\mathrm{SiC}$ is also proposed [37]. While the demonstrated Ge MSM detectors are able to achieve dark current suppression of two to four orders of magnitude, it is still an open question whether these MSM Ge photodetectors are competitive with PIN devices.

\subsection{Avalanche $P D$}

The simplest avalanche photodiode (APD) has a similar device structure to a $\mathrm{p}$-i-n photodiode. However, a voltage close to its breakdown is usually applied to APD for detection of low power signal with high sensitivity. Under sufficiently higher external bias, electrical field in the photodiode's depletion region becomes high enough to initiate impact ionization which is responsible for carrier multiplication. Therefore, one absorbed incoming photon does not only generate one electron/hole pair but rather a large number of EPHs leading to a quantum efficiency potentially large than unity. The most important performance indices for APD is excess noise factor quantified by effective ratio of electron and hole ionization rate $\left(\mathrm{k}_{\mathrm{eff}}\right)$, gain-efficiency product and sensitivity.

\subsection{Dark Current Criteria for Photodetectors}

An important issue in integrated photodetectors is dark current, which increases the power consumption of the receiver. Most importantly, shot noise associated with this leakage current undesirably degrades the Signal-to-Noise Ratio (SNR) leading to increased bit error rates (BERs).

Generally, dark currents less than $1 \mu \mathrm{A}$ are referred to as acceptable value for a high-speed receiver design, below which the transimpedance amplifier (TIA) noise is the main noise source [23,38,39]. In 
practice, a precise value of the required dark current depends upon the speed of operation and the amplifier design. In the recent successful demonstration of an Ge-on-Si photodetector-based receiver, photodetectors with dark current of both $\sim 10 \mathrm{nA}$ [38] and $\sim 2 \mu \mathrm{A}$ [24] were reported. Depending on the receiver design, a higher dark current level is tolerable with certain sacrifices in the receiver parameters. For example, Vivien et al. [40] have shown that with an increase of the input power of about $20 \%$ in comparison with photodetector without dark current, a photodetector with $300 \mu \mathrm{A}$ dark current is still able to ensure a BER of $10^{-18}$ at a frequency close to $50 \mathrm{GHz}$. The conclusion was drawn based on SPICE simulation taking into account of feedback resistance noise, the shot noise from detector dark current and photocurrent sources, and the transistor channel noise [41]. For the detailed modeling of the precise criteria for the dark current in high speed receiver, the readers are referred to [42] for further understanding.

\section{Ge Photodetector Light Coupling Schemes}

\subsection{Normal Incidence Photodetectors and the Bandwidth-Efficiency Tradeoff}

Normal incidence (NI) photodetectors are also known as vertical photodetectors or surface illuminated photodetectors. Normal incidence is the simplest light coupling scheme with incoming light illuminated on the top or bottom surface of the detector. Almost all the electrical structures, i.e., PIN, MSM and avalanche, can be fabricated in the fashion of NI photodetectors.

Figure 3. A Calculated carrier-transit-time-limiting bandwidth and efficiencies of normal incidence PIN Ge photodetector.

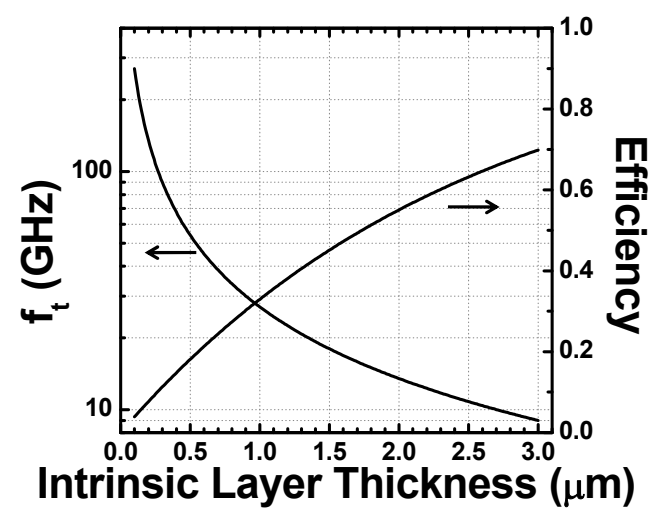

Due to its low process complexity, NI photodetectors are widely used in communication technologies. However, they suffer from an inherent drawback due to the bandwidth-efficiency tradeoff. This tradeoff results from the opposite requirement of the thickness of the photoabsorption layer for high bandwidth and high efficiency [43]. The carrier-transit-time-limiting bandwidth $f_{t}$ can be expressed as [44]:

$$
f_{t} \cong 0.45 \times \frac{v}{d}
$$

While the ideal efficiency $\eta$ assuming zero reflection and full carrier collection is:

$$
\eta \cong 1-e^{-\alpha \times d}
$$


where $v$ is carrier transit velocity, $\mathrm{d}$ is intrinsic region's thickness and $\alpha$ is material's absorption coefficient. Using $v=6 \times 10^{6} \mathrm{~cm} / \mathrm{s}$ for $\mathrm{Ge}$ and $\alpha=4,000 \mathrm{~cm}^{-1}$, the carrier-transit-time-limiting bandwidth and efficiencies versus intrinsic region thickness can be plotted as Figure 3. As can be seen, for a Ge device with $3 \mathrm{~dB}$ bandwidth of $100 \mathrm{GHz}$, an intrinsic layer thinner than $0.27 \mu \mathrm{m}$ is required with a resulting efficiency of $\sim 10 \%$.

\subsection{Resonant Cavity Enhanced (RCE) Detectors}

To overcome the tradeoff between bandwidth and efficiency in NI detectors, one method is to sandwich a thin layer of photo absorbing material between two light reflectors so that cavity resonance is enhanced $[45,46]$. In this structure, light is ideally trapped between the two reflectors and travels through the center light absorber multiple times until fully absorbed. At the same time, the photoabsorption layer can be thin enough to achieve high bandwidth. Another advantage of RCE detectors is the wavelength selectivity. When the light reflector is fabricated in the form of a Bragg reflector, only light in a small range of certain wavelengths is reflected effectively so as to produce high efficiency. The RCE device's light selectivity makes it especially useful for wavelength division multiplexing (WDM) systems.

Figure 4. Cross-sectional view of the back-illuminated Ge-SOI Schottky photodetector. From [45].

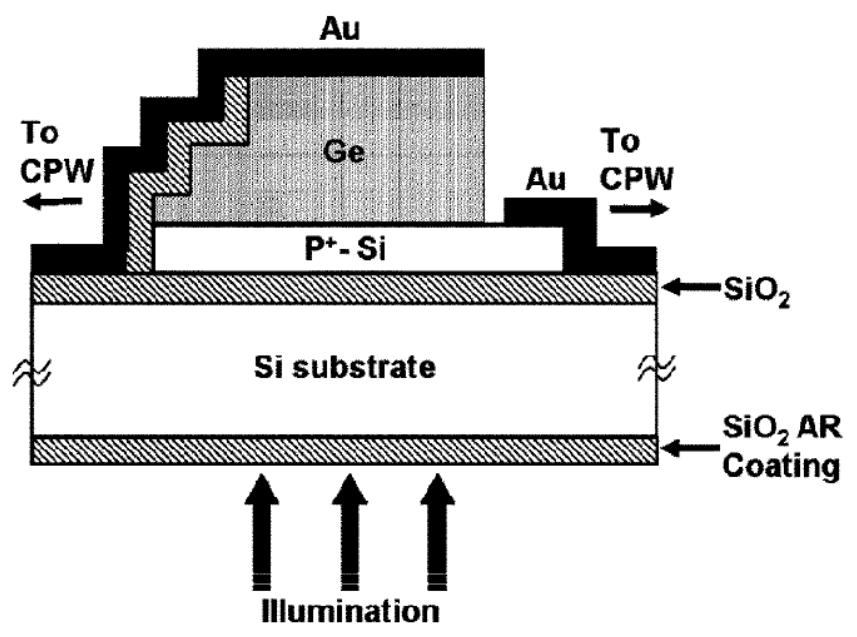

Ge RCE Schottky photodetectors (Figure 4) were demonstrated by Dosunmu et al. [45] in 2005. The resonant cavity was formed between the Au reflecting top metal contact and the SOI substrate. The backside of the SOI wafer was polished to facilitate light coupling. Schottky contact was formed between the top contact $\mathrm{Au}$ and the Ge layer while the bottom contact of $\mathrm{Au}$ and $\mathrm{p}+$-Si was ohmic contact. The resonant wavelength was found at around $1,538 \mathrm{~nm}$, leading to an increased quantum efficiency of $59 \%$.

Although RCE photodetectors solve the bandwidth-efficiency tradeoff to some extent, the fabrication of high reflectivity mirrors increase the design and process complexity significantly. The multiple layers needed for effective reflection also make RCE detectors difficult integrate with other functional devices. Therefore, other methods with more process and integration friendliness are still required. 


\subsection{Waveguide Photodetectors}

Waveguide integrated photodetectors have been considered to be one of the most promising candidates for overcoming the bandwidth-efficiency tradeoff in normal incidence detectors. In this configuration, a light signal is delivered to the device by in-plane optical waveguide rather than top down, permitting the bandwidth and efficiency to be determined almost independently because the efficiency is specified no longer by the photoabsorption layer thickness, but rather by the waveguide length. Furthermore, large scale integration of Si optical and electrical devices requires all devices to be fabricated on the same planar wafer, which makes an optical waveguide indispensable. Thus integration of a waveguide with photodetectors seems to be a natural choice. The development of Ge-on-Si photodetectors has been going on for more than ten years. In Table 2 and Figure 5, performances reported for some typical Ge photodetectors are summarized.

Table 2. Summary of performances from selected Ge photodetectors.

\begin{tabular}{|c|c|c|c|c|c|c|c|}
\hline Year & Reference & 1st author & structure & $\begin{array}{c}\mathbf{I}_{\mathrm{dark}}(\boldsymbol{\mu A}) @ \\
-1 \mathrm{~V}\end{array}$ & $\begin{array}{l}\text { Responsivity } \\
@ \lambda=1.55 \mu \mathrm{m} \\
@-1 \mathrm{~V}(\mathrm{~A} / \mathrm{W})\end{array}$ & $\begin{array}{c}\text { Highest } 3 \mathrm{~dB} \\
\text { electrical bandwidth } \\
(\mathrm{GHz}) \\
\end{array}$ & $\begin{array}{c}\text { APD } \\
\text { gain-bandwidth } \\
\text { product }(\mathrm{GHz}) \\
\end{array}$ \\
\hline 2000 & {$[4]$} & L. Colace & NI PIN & 12 & 0.25 & $\sim 0.4 @-4 \mathrm{~V}$ & - \\
\hline 2002 & [17] & S. Fama & NI PIN & 1.2 & 0.75 & 2.5@-1 V & - \\
\hline 2005 & [14] & M. Jutzi & NI PIN & 0.08 & 0.035@0 V & 39@-2 V & - \\
\hline 2005 & [33] & J. Liu & NI PIN & $\sim 0.8$ & 0.56 & 8.5@-1 V & - \\
\hline 2005 & {$[45]$} & O. I. Dosunmu & NI PIN RCE & $0.38 @-5 \mathrm{~V}$ & 0.73 & 12.1@-3 V & - \\
\hline 2006 & {$[18]$} & L. Colace & NI PIN & $\sim 10$ & 0.2 & 10@-1 V & - \\
\hline 2007 & {$[40]$} & L. Vivien & WG MSM & 130 & 1 & 25@-6 V & - \\
\hline 2007 & [23] & D. Ahn & WG PIN & 0.9 & 0.87 & 7.5@-3 V & - \\
\hline 2007 & [47] & T. Yin & WG PIN & $0.267 @-2 \mathrm{~V}$ & $1.16 @-2 \mathrm{~V}$ & $29.4 @-2 \mathrm{~V}$ & - \\
\hline 2008 & [48] & J. Wang & WG PIN & 0.06 & 0.65 & 18@-1 V & - \\
\hline 2009 & [49] & L. Chen & WG MSM & 4@-5 V & $\sim 1$ & 40@-5 V & - \\
\hline 2009 & [50] & S. Klinger & NI PIN & $\sim 0.10$ & $0.05 @-2 \mathrm{~V}$ & 49@-2 V & - \\
\hline 2009 & [25], & D. Suh & NI PIN & 0.042 & 0.47 & 36@-3 V & - \\
\hline 2009 & [20] & L. Vivien & WG PIN & $\sim 1$ & 1 & 42@-4 V & - \\
\hline 2009 & {$[51]$} & D. Suh & WG PIN & 0.072 & 0.8 & 47@-3 V & - \\
\hline 2010 & [19] & D. Feng & WG PIN & 1.3 & 1.1 & $36.8 @-3 \mathrm{~V}$ & - \\
\hline 2010 & {$[52]$} & S. Assefa & WG MSM & 90 & 0.14 & 40@-2 V & - \\
\hline 2008 & [53] & Y. Kang & NI SACM APD & $\sim 10 @ 25 \mathrm{~V}$ & 15.6@ 25 V@1.3 um & $\sim 12 @ \sim 25 \mathrm{~V}$ & 340 \\
\hline 2010 & [54] & K. Ang & WG SACM APD & 100@23 V & 16.8@ 23 V & 5@ 23 V & 105 \\
\hline 2010 & [55] & S. Assefa & WG MSM APD & 100@ & - & 35@1.5 V & 350 \\
\hline
\end{tabular}


Figure 5. Bandwidth and responsivity of selected Ge photodetectors.

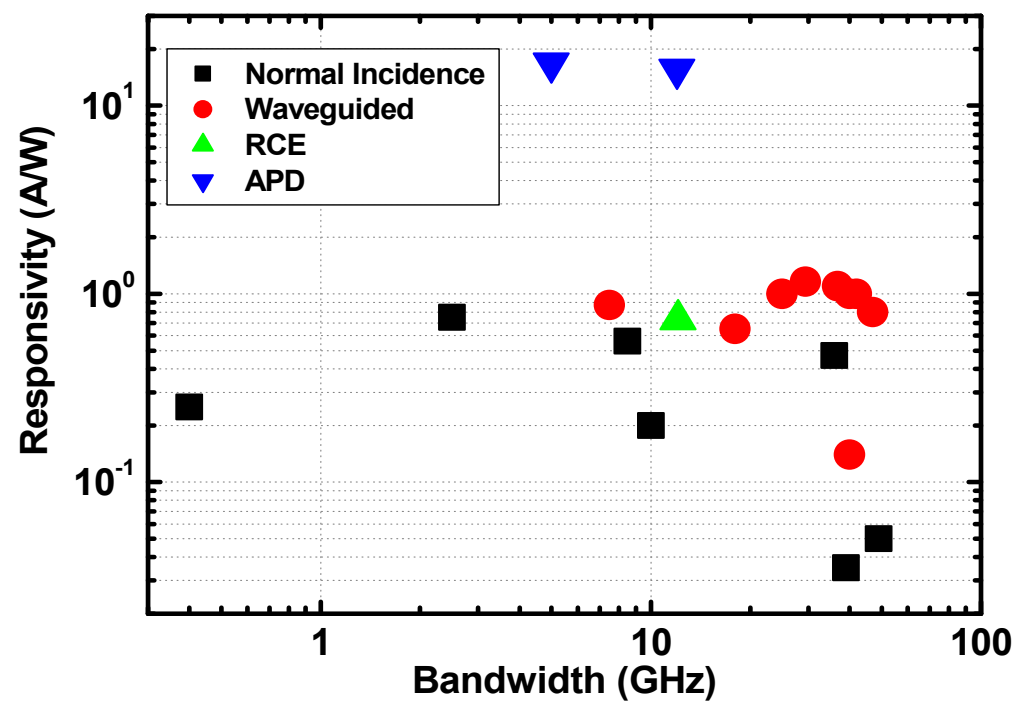

\section{Research Trends in Ge Photodetectors}

In this section, historical research trends are identified and described. The remaining technical issues and future directions are also discussed here.

\subsection{Normal Incidence to Waveguide Integration}

At the starting stage of Ge-on-Si photodetector development for Si photonics applications, normal incidence Ge photodetectors were first fabricated and comprehensively studied $[4,14,17,18,22,33,50,53,56-64]$ due to their ease of processing.

Figure 6. 3D view of the PIN Ge photodiode with $49 \mathrm{GHz}$ bandwidth. From [50].

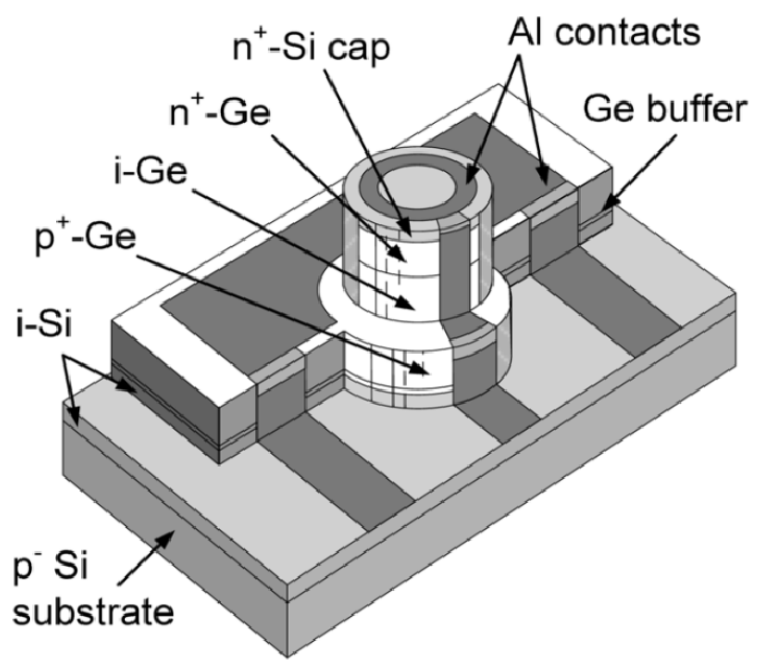

Due to the bandwidth-efficiency tradeoff, typical NI incidence Ge photodetectors offer moderate quantum efficiencies and bandwidths. Among the reported NI photodetectors, Klinger et al. [50] reported the highest bandwidth of $49 \mathrm{GHz}$ for Ge-based photodetectors. The Ge p-i-n photodiode was fabricated in Ge grown by MBE two-step Ge growth (Figure 6). Given the nominal Ge intrinsic layer 
thickness of $300 \mathrm{~nm}$, detector diameter of $10 \mu \mathrm{m}$ and the series resistance of $25 \Omega$, the theoretical bandwidth of $54.3 \mathrm{GHz}$ corresponds well with the experimental value. It should be noted that the reported improved $3 \mathrm{~dB}$ frequency of $49 \mathrm{GHz}$ from previous result $(39 \mathrm{GHz})$ [14] is mainly due to the reduced series resistance (Rs) of $15 \Omega$ from $32 \Omega$. The reported responsivity at $1,550 \mathrm{~nm}$ is $\sim 0.05 \mathrm{~A} / \mathrm{W}$ limited by small device footprint and relatively large density of defects in the Ge layer.

In terms of high responsivity, a thick Ge absorption layer is need. The highest reported value at $1,550 \mathrm{~nm}$ wavelength for NI photodetector is $0.75 \mathrm{~A} / \mathrm{W}$ from a PIN diode with $\sim 4 \mu \mathrm{m}$ thick Ge layer fabricated and reported by Fama and coworkers [17]. The Ge layer was epitaxially grown on a Si substrate by two-step UHVCVD combined with high temperature cyclic annealling for the reduction of dislocation density. A time response of less than $200 \mathrm{ps}$ and operation $>2.5 \mathrm{~Gb} / \mathrm{s}$ was also demonstrated.

The study of the Ge normal incidence photodetectors provides valuable insights into the $\mathrm{Ge} / \mathrm{Si}$ system and its properties. As the Ge growth technique becomes mature and the particulars of $\mathrm{Ge} / \mathrm{Si}$ devices have been studied in detail, research has gradually been redirected to the integration of Ge photodetectors on $\mathrm{Si}$ waveguides to decouple the tradeoff between bandwidth and efficiency mentioned above. Since Si photonics also require devices to be monolithically integrated on the same Si substrate using an on-wafer optical waveguide, integration of Ge photodetectors with waveguides seems mandatory.

To date, a number of groups have demonstrated waveguided Ge photodetectors, including MIT [1,23,65-67], IEF [20,40], IME [35,48,54,68,69], INTEL [47,70], IBM [52,55,71] and Kotura [19,72]. Both PIN and MSM structures are reported in these waveguide photodetectors with comparable performance and high speeds of around $40 \mathrm{GBit} / \mathrm{s}$.

\subsection{Improvement of Speed Performance of Waveguide Ge Photodetectors}

Another trend of the continuous evolvement of Ge photodetectors is the increase of detector bandwidth. At the starting point of Ge detectors' integration with Si waveguides, Ge growth on SOI wafers and optical coupling between Ge detectors and Si optical waveguides was first explored. The reported detectors are $\sim 100 \mu \mathrm{m}$ long to ensure full absorption of light around $1.55 \mu \mathrm{m}$ wavelength, inevitably leading to large device capacitance so that the bandwidth are limited $<10 \mathrm{GHz}$ by RC delay.

Nowadays, special care in design is given to detector's bandwidth performance. With adoption of shorter devices, sophisticated radio-frequency coplanar waveguides (CPW), metal interconnections and frequency measurement technologies, $40 \mathrm{Gbit} / \mathrm{s}$ operation was reported by several groups [47,49,52,73], with waveguide detector bandwidths as high as $47 \mathrm{GHz}$ [73].

\subsection{Zero-Bias PIN Photodiode}

As discussed in Section 3.4, high dark current leads to high stand-by power consumption in addition to the degraded SNR. Moreover, it is desirable for the detector and the receiver circuit to operate on a single power supply which often restricts the bias voltage for photodetector to be less than 1.5 $\mathrm{V}$ [74]. As a result, there has been an increasing research interest in the development of low-bias or even zero bias PIN photodiodes.

In terms of responsivity, Liu et al. [33] measured their Ge PIN detector's responsivity over the wide spectrum of $650 \mathrm{~nm}-1,650 \mathrm{~nm}$. The reported responsivity at $0 \mathrm{~V}$ bias is more than $98 \%$ of the saturated 
value at $2 \mathrm{~V}$ reverse bias, which was attributed to high carrier collection efficiency resulting from the high built-in electric field in the diode's depletion region. High speed operation at zero-bias was demonstrated by Jutzi et al. [14]. From PIN Ge detectors with diameter of $10 \mu \mathrm{m}$, a record high zero-bias $3 \mathrm{~dB}$ bandwidth of $25.1 \mathrm{GHz}$ was obtained (see Figure 7).

Figure 7. Normalized frequency response at a wavelength of $1,552 \mathrm{~nm}$ for PIN detectors with diameters varying from 10 to $30 \mu \mathrm{m}$. From [14].

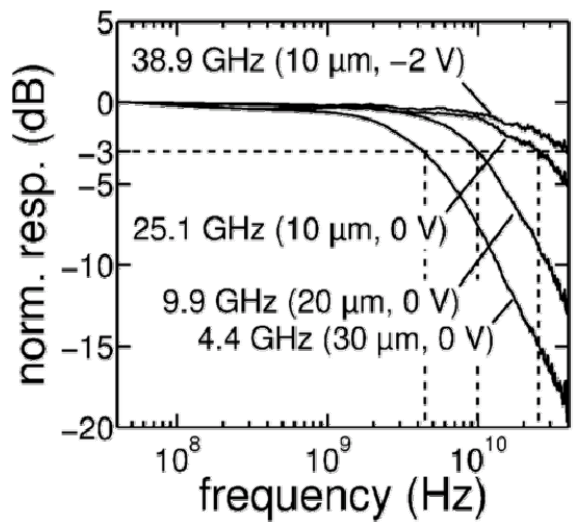

\subsection{Avalanche Photodetectors}

For Ge photodetectors' application in Si photonics IC, a next level pre-amplifier is necessary to further transform the current signal into a voltage signal for later CMOS IC processing. Avalanche photodetectors offers much lower signal-to-noise ratio compared to PIN or MSM structures. Therefore, more and more interest is being focused on Ge-based avalanche photodetectors. Recently, Intel [53], IBM [55] and IME [54] have all reported successful fabrication of such devices.

The first Ge-based APD was demonstrated by Kang et al. [53]. For the reported device, a separate-absorption-charge-multiplication (SACM) configuration (Figure 8) is used to take advantage of both Si's low noise property and Ge's strong absorption near $1.55 \mu \mathrm{m}$ wavelength. The device exhibits low excess noise with low $\mathrm{k}_{\mathrm{eff}}$ of $\sim 0.09$. The reported sensitivity of $-28 \mathrm{dBm}$ at $10 \mathrm{~Gb} / \mathrm{s}$ is equivalent to a commercial III-V APD and the bandwidth-efficiency product of $340 \mathrm{GHz}$ is even higher than its III-V counterpart, thanks to much lower k value of Si compare to InP material.

Figure 8. Schematic of Ge/Si APD with a typical SACM configuration. From [53].

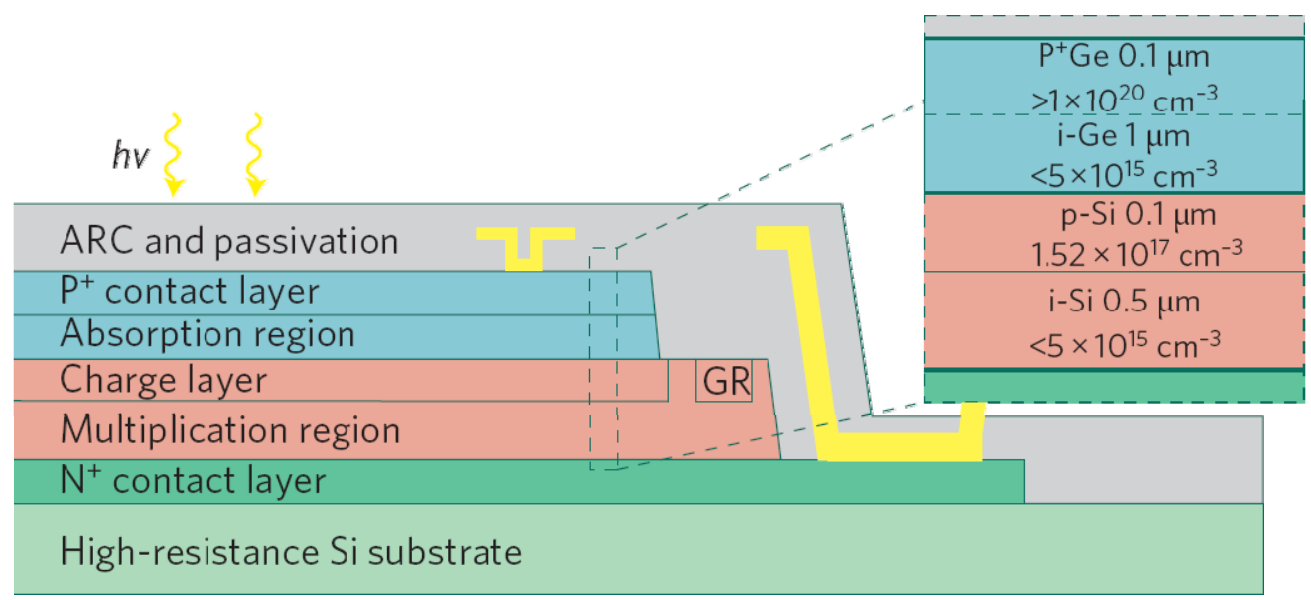


Another Ge APD configuration is a conventional MSM structure with nano-engineered metal-to-metal spacing as small as $200 \mathrm{nn}$ reported by Assefa et al. [55]. With a low bias voltage of $\sim 1.5 \mathrm{~V}$, the electric field in the immediate vicinity of the metal contact is already high enough to initiate avalanche amplification. Although the whole APD structure is built on Ge, whose properties are not optimized for APD, the device exhibits a excess noise factor with $\mathrm{k}_{\mathrm{eff}} \sim 0.2$, high speed of $\sim 40 \mathrm{GHz}$, and bandwidth-efficiency product of $350 \mathrm{GHz}$ at the wavelength of $1.3 \mu \mathrm{m}$. Although the high dark current due to the small metal spacing requires more optimization, the Ge MSM APD shows great potential for the application of $\mathrm{Ge}$ in avalanche photodetection.

The APD devices reported above are working at $1.3 \mu \mathrm{m}$ due to the incorporation of Si into $\mathrm{Ge}$ which gives rise to undesired reduction of the absorption efficiency at 1,550 nm. Using two-step Ge growth with a SiGe buffer layer method, the first waveguide-base Ge APD working at $1.55 \mu \mathrm{m}$ was reported by Ang et al. [54]. The device is fabricated based on a SACM structure. A waveguide was used to increase device efficiency and facilitate future $\mathrm{Si}$ photonics integration. The reported high responsivity at unity gain was as high as $\sim 0.8 \mathrm{~A} / \mathrm{W}$ and a product bandwidth-efficiency of $105 \mathrm{GHz}$ was achieved.

\subsection{Remaining Technical Issues and Future Research Trends}

\subsubsection{Pursuit of Higher Bandwidth}

Nowadays, the reported Ge photodetectors' bandwidths are approaching $50 \mathrm{GHz}$, ready for near-future $40 \mathrm{~Gb} / \mathrm{s}$ applications. On the other hand, in correspondence with III-V photodetectors, it can be seen that there is still much room for enhancement. For bandwidths beyond $100 \mathrm{GHz}$, much thinner Ge intrinsic layers should be used. As in the high frequency region, undesirable parasitic effect such as contact resistance, stray capacitance and inductance may become the main limiting factors in bandwidth performance. Given the fact that reducing the intrinsic region's thickness for smaller carrier transition time at the same time leads to increase of device capacitance, the mushroom-mesa structure [43] (Figure 9) may be of help for further bandwidth evolution, since it is capable of reducing the Rs and capacitance simultaneously.

Figure 9. Schematic view of III-V photodetector based on mushroom structure. From [43].

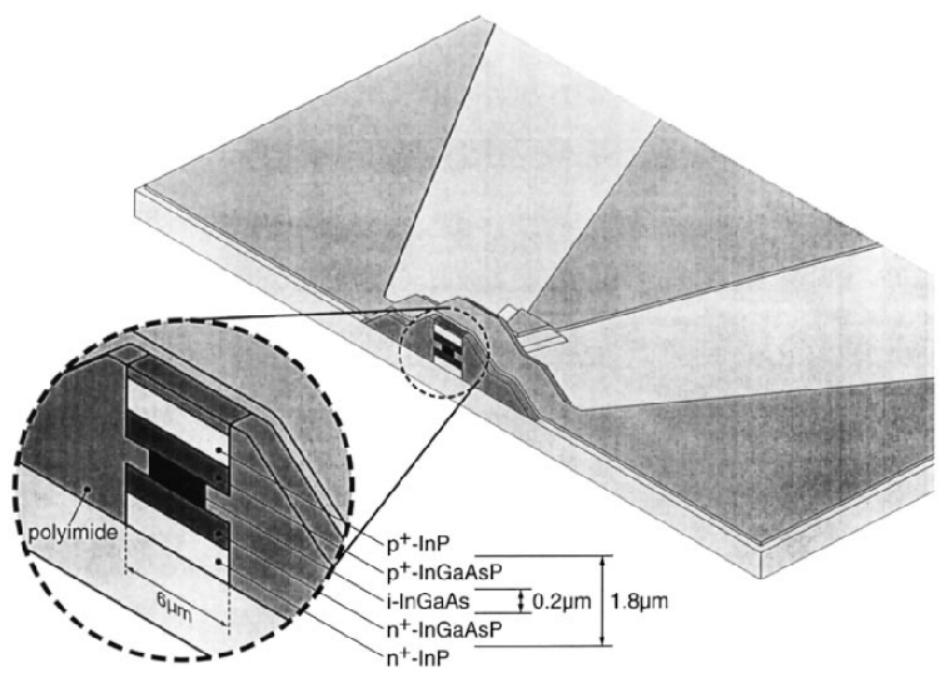




\subsubsection{Monolithic Integration of Ge Photodetectors with CMOS Integrated Circuits}

Essential for future Si EPIC is the co-integration of Ge photodetectors with functional CMOS circuits, which brings optical detection and further signal processing together. Therefore, there has been much effort in pursuing such integration. However, fabrication of high performance $\mathrm{Ge}$ photodetectors together with conventional CMOS devices comes with several technical issues that must be addressed, including the thermal budget issue, the cross contamination issues and the non-planarity issue due to Ge layer thickness.

To avoid the high temperatures needed during two-step Ge growth and subsequent cyclic annealling for dislocation density reduction, thermal evaporation polycrystalline Ge with full compatibility with CMOS fabrication due to its deposition temperature as low as $\sim 300{ }^{\circ} \mathrm{C}$ was demonstrated [6]. However, the relatively inferior electrical quality of the Ge limits the performance of the photodetector making this technique not suitable for high-speed high efficiency Si EPIC for future data transmission.

Another study [38] uses a wire-bonding technique to connect separately-manufactured Ge photodetectors and a Si CMOS amplifier (see Figure 10). While this approach reduces the process complexity and cost considerably, undesirable large parasitic capacitance from the bonding pad and inductance from the bonding wire inevitably sacrifice the speed performance.

Figure 10. Optical micrograph showing a Ge-on-SOI photodiode wire-bonded to a high-gain CMOS IC. High-magnification optical and SEM micrographs of the photodiode are also shown. From [38].

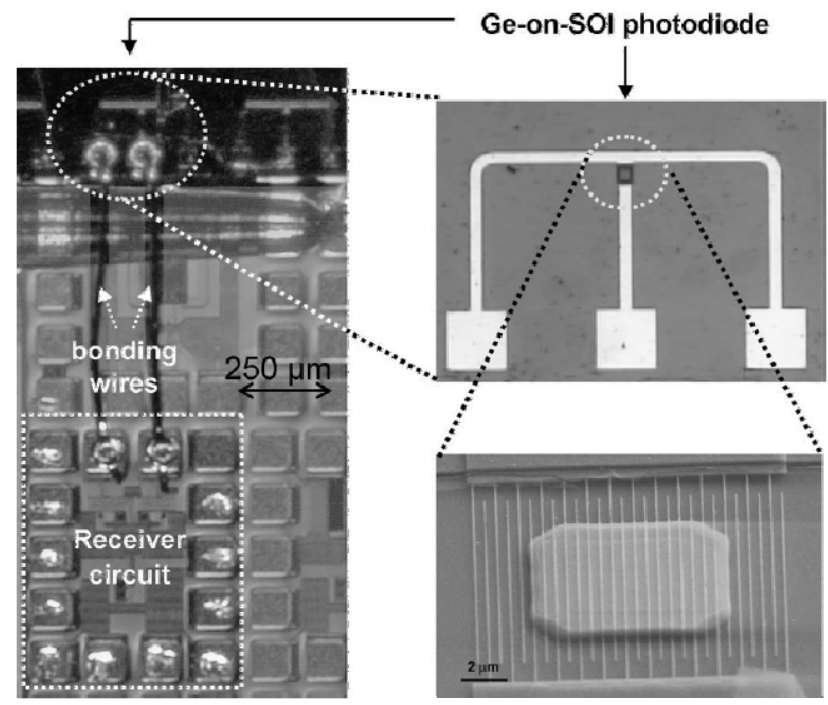

Masini et al. [24] reported the first successful monolithic integration of a Ge photodetector with CMOS on the same wafer (Figure 11). Special attention was given to the thermal budget planning in the process design. Since high temperature is compulsory for gate oxide growth, Ge grow was inserted after the gate processing. Furthermore, to avoid damage by high temperature Ge layer epitaxy, reduced-press chemical vapor deposition (RPCVD) at $350{ }^{\circ} \mathrm{C}$ was used. Also, no high temperature annealling was carried out. To address the non-planarity issue normally induced by thick Ge of several microns used for efficient light absorption, a waveguided photodetector with a thin Ge layer of $\sim 200 \mathrm{~nm}$ was used. Since with low temperature Ge growth and no curing annealling, the 
fabricated $25 \mu \mathrm{m}$-long detector demonstrated large dark current of $10 \mu \mathrm{A} @-1 \mathrm{~V}$ and relatively low responsivity of $\sim 0.6 \mathrm{~A} / \mathrm{W}$ at $1,554 \mathrm{~nm}$.

Figure 11. A four-channel, $10 \mathrm{Gbps}$ monolithic optical receiver in $130 \mathrm{~nm}$ CMOS with integrated Ge waveguide photodetectors. From [24].

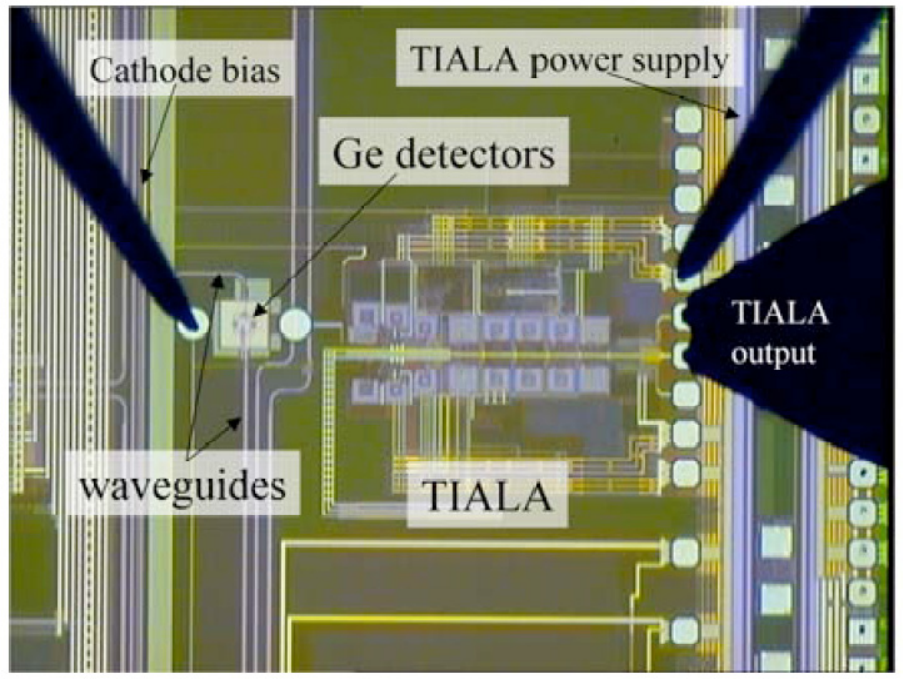

Figure 12. (a) Schematic showing the "electronic-first and photonic-last" integration approach for monolithically fabricating the Ge p-i-n photodetector and Si CMOS circuit on common SOI platform. (b) Comparable threshold voltages were observed in both n-MOS and p-MOS transistors despite the introduction of an additional thermal budget during the Ge epitaxy growth. From [75].
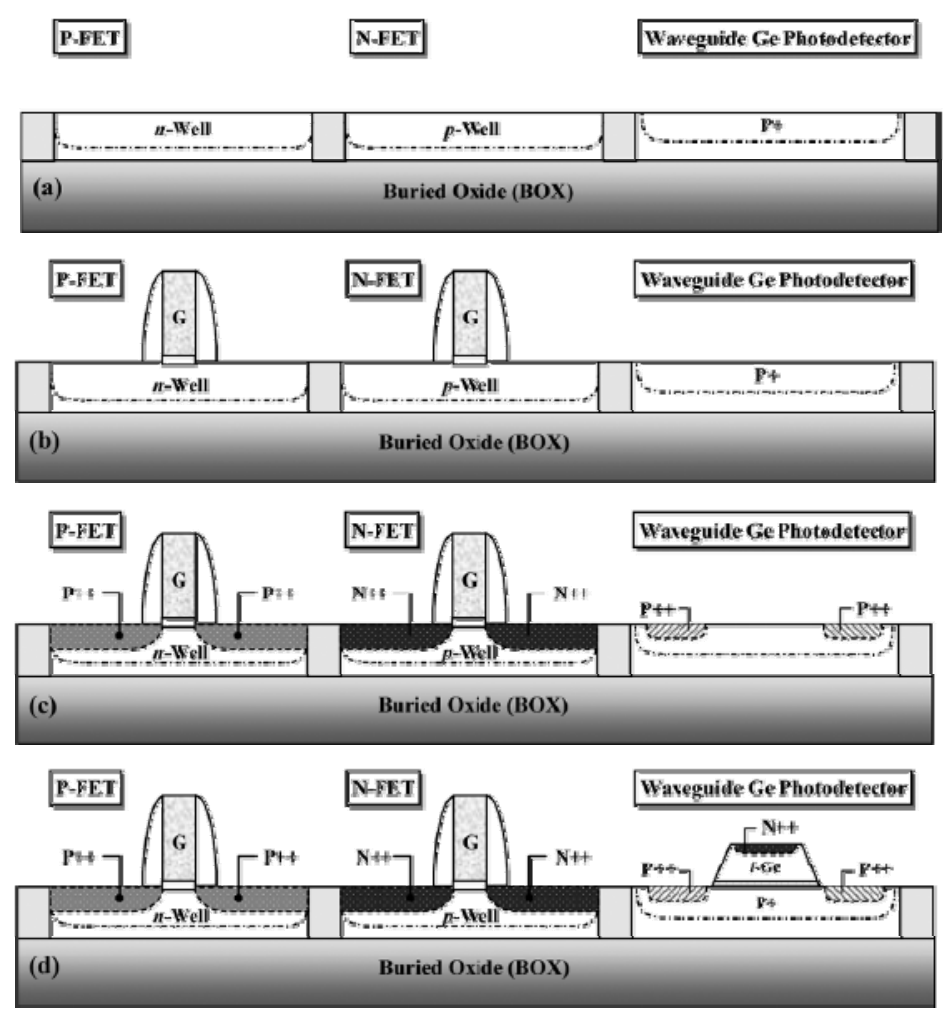

(a)

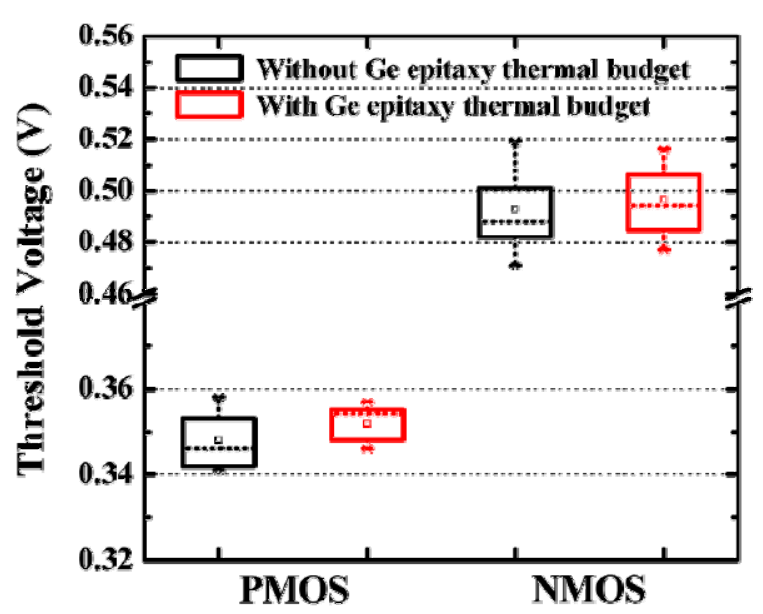

(b) 
The impact of high temperature prebake treatment before Ge epitaxy on the monolithic integration was studied by Ang et al. [75]. For UHVCVD Ge epitaxy, an $800{ }^{\circ} \mathrm{C}$ prebake before actual growth is usually necessary for removal of native oxide from Si surface to ensure Ge epitaxy quality. However, such high temperature causes thermally enhanced dopant diffusion in CMOS devices leading to possible shift in transistor's threshold voltage $\left(\mathrm{V}_{\text {th }}\right)$. This concern is especially the case for short channel devices since the impact of dopant diffusion is larger. It was found that for transistors with effective channel length of $\sim 180 \mathrm{~nm}$, the additional $800{ }^{\circ} \mathrm{C}$ thermal budget virtually have no apparent impact on the $\mathrm{V}_{\text {th }}$ (Figure 12(b)). However, for the state-of-the-art aggressively scaled down devices with critical dimension well below $100 \mathrm{~nm}$, whether the influence of the $800{ }^{\circ} \mathrm{C}$ prebake is still negligible leaves an open question. As a result, research and development of low temperature Ge growth technique with low dislocation density Ge film would be expected to be one of the most focused directions for future research. The reported monolithic integration scheme is also based on "electronic-first and photonic-last" approach (Figure 12(a)) to avoid Ge degradation and cross contamination during the high temperature gate oxidation and dopant activation in Si transistors.

\subsubsection{Plasmonics for Extreme Light Concentration}

For higher speed, lower noise and suppressed power consumption, photodetectors are being fabricated in smaller dimensions [76]. However, previously the physical dimensions of the photodetectors were limited in the micrometer range by classical diffraction theory.

Recently, the amazing ability of plasmonic structures to concentrate light both laterally and in the depth of a semiconductor material beyond the diffraction limit into the deep-subwavelength-dimension was reported by Ishi et al. [77] A concentric grating surface plasmon antenna of $10 \mu \mathrm{m}$ diameter was demonstrated to concentrate light into the center $\mathrm{Si}$ mesa Schottky diode of an active area of $300 \mathrm{~nm}$ in diameter (Figure 13). The observed more than 20-fold enhancement in photocurrent confirms the plasmonic effect. The estimated bandwidth of such small detector exceeds $100 \mathrm{GHz}$.

Because of its promise in Ge photodetector's drastic miniaturization into the nano-scale domain and expected high speed, plasmonics technology's application in Ge-based detectors are being actively pursued both theoretically [78] and experimentally [79] making it one of the major research directions in the future.

Figure 13. Concentric grating coupler used in the nano-photodiode. From [77].

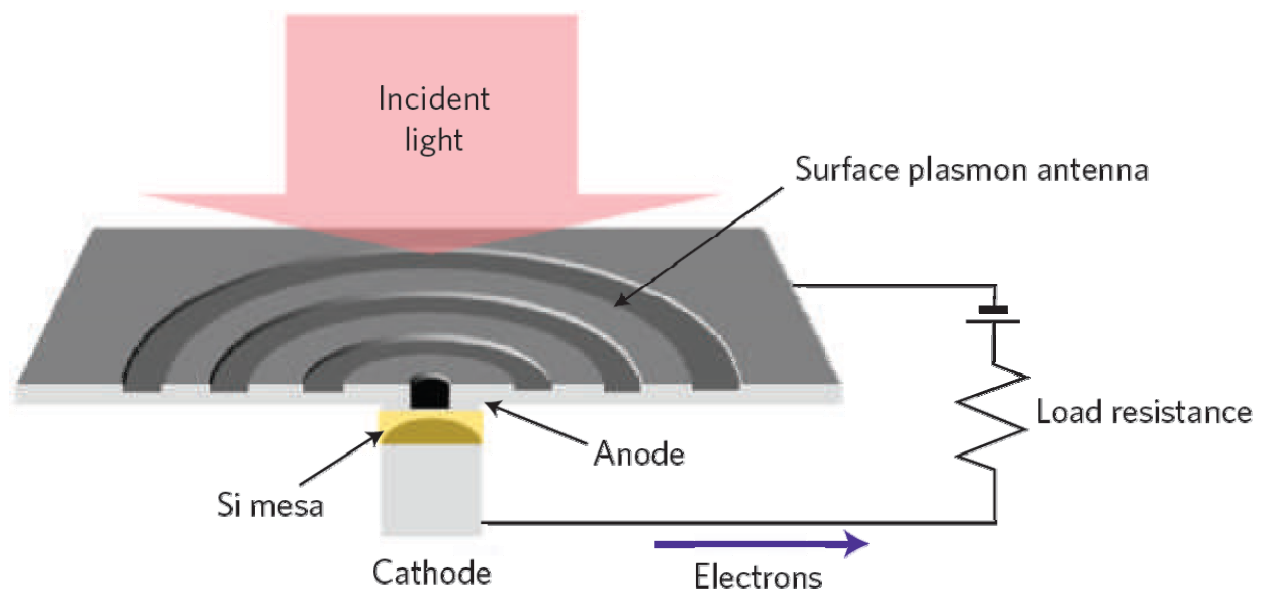




\section{Conclusions}

This paper summarizes the historical and current trends of Ge-on-Si photodetectors development, which is essential for Si EPIC integration. Various electrical structures (PIN, MSM, and avalanche) and optical coupling schemes (normal incidence, resonant cavity enhancement and waveguide integration) have been adopted in Ge photodetectors, demonstrating high responsivity approaching 100\% quantum efficiency and high speed operation at around $40 \mathrm{~Gb} / \mathrm{s}$. With practical $\mathrm{Si}$ photonics EPIC around the corner, higher speed, easier integration with CMOS fabrication and novel approaches such as plasmonics-enabled nano-scale detector will become the main focuses of research in the near future.

\section{References}

1. Michel, J.; Liu, J.; Ahn, D.; Sparacin, D.; Sun, R.; Hong, C.; Giziewicz, W.; Beals, M.; Kimerling, L.; Kopa, A. Advances in fully CMOS integrated photonic devices. Proc. SPIE 2007, doi:10.1117/12.706586.

2. Liu, J.; Sun, X.; Camacho-Aguilera, R.; Kimerling, L.; Michel, J. Ge-on-Si laser operating at room temperature. Optic. lett. 2010, 35, 679-681.

3. Liu, J.; Beals, M.; Pomerene, A.; Bernardis, S.; Sun, R.; Cheng, J.; Kimerling, L.; Michel, J. Waveguide-integrated, ultralow-energy GeSi electro-absorption modulators. Nat. Photon. 2008, 2 , 433-437.

4. Colace, L.; Masini, G.; Assanto, G.; Luan, H.; Wada, K.; Kimerling, L. Efficient high-speed near-infrared Ge photodetectors integrated on Si substrates. Appl. Phys. Lett. 2000, 76, 1231-1233.

5. Luryi, S.; Kastalsky, A.; Bean, J. New infrared detector on a silicon chip. IEEE Trans. Electron. Dev. 1984, 31, 1135-1139.

6. Masini, G.; Colace, L.; Galluzzi, F.; Assanto, G. Advances in the field of poly-Ge on Si near infrared photodetectors. Mater. Sci. Eng. B 2000, 69, 257-260.

7. Masini, G.; Cencelli, V.; Colace, L.; De Notaristefani, F.; Assanto, G. Monolithic integration of near-infrared $\mathrm{Ge}$ photodetectors with $\mathrm{Si}$ complementary metal-oxide-semiconductor readout electronics. Appl. Phys. Lett. 2002, 80, 3268-3270.

8. Colace, L.; Masini, G.; Cencelli, V.; DeNotaristefani, F.; Assanto, G. A near-infrared digital camera in polycrystalline germanium integrated on silicon. IEEE J. Quantum Electron. 2007, 43, 311-315.

9. Sorianello, V.; Balbi, M.; Colace, L.; Assanto, G.; Socci, L.; Bolla, L.; Mutinati, G.; Romagnoli, M. Guided-wave photodetectors in germanium on SOI optical chips. Physica E 2009, 41, 1090-1093.

10. Fitzgerald, E.; Xie, Y.; Green, M.; Brasen, D.; Kortan, A.; Michel, J.; Mii, Y.; Weir, B. Totally relaxed GeSi layers with low threading dislocation densities grown on Si substrates. Appl. Phys. Lett. 1991, 59, 811-814.

11. Fan, J.; Tsaur, B.; Gale, R.; Davis, F. Reducing Dislocations in Semiconductors Utilizing Repeated Thermal Cycling during Multistage Epitaxial Growth. U.S. Patent 4,632,712, 30 December 1986. 
12. Colace, L.; Masini, G.; Galluzzi, F.; Assanto, G.; Capellini, G.; Di Gaspare, L.; Palange, E.; Evangelisti, F. Metal-semiconductor-metal near-infrared light detector based on epitaxial Ge/Si. Appl. Phys. Lett. 1998, 72, 3175-3178.

13. Hartmann, J.M.; Abbadie, A.; Papon, A.M.; Holliger, P.; Rolland, G.; Billon, T.; Fedeli, J.M.; Rouviere, M.; Vivien, L.; Laval, S. Reduced pressure - chemical vapor deposition of Ge thick layers on $\mathrm{Si}(001)$ for 1.3-1.55-mu m photodetection. J. Appl. Phys. 2004, 95, 5905-5913.

14. Jutzi, M.; Berroth, M.; Wohl, G.; Oehme, M.; Kasper, E. Ge-on-Si vertical incidence photodiodes with 39-GHz bandwidth. IEEE Photon. Technol. Lett. 2005, 17, 1510-1512.

15. Luan, H.; Lim, D.; Colace, L.; Masini, G.; Assanto, G.; Wada, K.; Kimerling, L. Germanium Photodetectors for Silicon Microphotonics by Direct Epitaxy on Silicon; Warrendale, P., Ed.; Materials Research Society: Warrendale, PA, USA, 2000; pp. 279-284.

16. Luan, H.; Lim, D.; Lee, K.; Chen, K.; Sandland, J.; Wada, K.; Kimerling, L. High-quality Ge epilayers on Si with low threading-dislocation densities. Appl. Phys. Lett. 1999, 75, 2909-2912.

17. Fama, S.; Colace, L.; Masini, G.; Assanto, G.; Luan, H. High performance germanium-on-silicon detectors for optical communications. Appl. Phys. Lett. 2002, 81, 586-589.

18. Colace, L.; Balbi, M.; Masini, G.; Assanto, G.; Luan, H.; Kimerling, L. Ge on Si pin photodiodes operating at $10 \mathrm{Gbit} / \mathrm{s}$. Appl. Phys. Lett. 2006, 88, 101111-101114.

19. Feng, N.; Dong, P.; Zheng, D.; Liao, S.; Liang, H.; Shafiiha, R.; Feng, D.; Li, G.; Cunningham, J.; Krishnamoorthy, A. Vertical pin germanium photodetector with high external responsivity integrated with large core Si waveguides. Opt. Express 2010, 18, 96-101.

20. Vivien, L.; Osmond, J.; Fédéli, J.; Marris-Morini, D.; Crozat, P.; Damlencourt, J.; Cassan, E.; Lecunff, Y.; Laval, S. $42 \mathrm{GHz}$ pin Germanium photodetector integrated in a silicon-on-insulator waveguide. Opt. Express 2009, 17, 6252-6257.

21. Morse, M.; Dosunmu, O.; Sarid, G.; Chetrit, Y. Performance of Ge-on-Si pin photodetectors for standard receiver modules. IEEE Photon. Technol. Lett. 2006, 18, 2442-2444.

22. Dehlinger, G.; Koester, S.; Schaub, J.; Chu, J.; Ouyang, Q.; Grill, A.; Technologie, I.; Villach, A. High-speed germanium-on-SOI lateral PIN photodiodes. IEEE Photon. Technol. Lett. 2004, 16, 2547-2549.

23. Ahn, D.; Hong, C.; Liu, J.; Giziewicz, W.; Beals, M.; Kimerling, L.; Michel, J.; Chen, J.; Krtner, F. High performance, waveguide integrated Ge photodetectors. Opt. Express 2007, 15, 3916-3921.

24. Masini, G.; Capellini, G.; Witzens, J.; Gunn, C. A 1,550nm, 10 Gbps monolithic optical receiver in $130 \mathrm{~nm}$ CMOS with integrated Ge waveguide photodetector. In Proceedings of the 4th IEEE International Conference on Group IV Photonics 2007, Tokyo, Japan, 19-21 September 2007; pp. 1-3.

25. Suh, D.; Kim, S.; Joo, J.; Kim, G. 36-GHz high-responsivity Ge photodetectors grown by RPCVD. IEEE Photon. Technol. Lett. 2009, 21, 672-674.

26. Huang, Z.; Oh, J.; Campbell, J. Back-side-illuminated high-speed Ge photodetector fabricated on Si substrate using thin SiGe buffer layers. Appl. Phys. Lett. 2004, 85, 3286-3289.

27. Nakatsuru, J.; Date, H.; Mashiro, S.; Ikemoto, M. Growth of High Quality Ge Epitaxial Layer on Si (100) Substrate Using Ultra Thin SiGe Buffer; Materials Research Society: Warrendale, PA, USA, 2006; pp. 315-320. 
28. Loh, T.; Nguyen, H.; Tung, C.; Trigg, A.; Lo, G.; Balasubramanian, N.; Kwong, D.; Tripathy, S. Ultrathin low temperature SiGe buffer for the growth of high quality Ge epilayer on Si (100) by ultrahigh vacuum chemical vapor deposition. Appl. Phys. Lett. 2007, 90, 092108:1-092108:3.

29. Choi, D.; Ge, Y.; Harris, J.S.; Cagnon, J.; Stemmer, S. Low surface roughness and threading dislocation density Ge growth on Si (0 0 1). J. Cryst. Growth 2008, 310, 4273-4279.

30. Osmond, J.; Isella, G.; Chrastina, D.; Kaufmann, R.; Acciarri, M.; Kanel, H.V. Ultralow dark current $\mathrm{Ge} / \mathrm{Si}(100)$ photodiodes with low thermal budget. Appl. Phys. Lett. 2009, 94, 201106:1-201106:3.

31. Zang, H.; Loh, W.; Ye, J.; Loh, T.H.; Lo, G.; Cho, B. Integration of dual channels MOSFET on defect-free, tensile-strained germanium on Silicon. In Proceedings of International Conference on Solid State Devices and Materials (SSDM 2007), Ibaraki, Japan, 18-21 September 2007; pp. 32-33.

32. Wang, J.; Loh, W.Y.; Zang, H.; Yu, M.B.; Chua, K.T.; Loh, T.H.; Ye, J.D.; Yang, R.; Wang, X.L.; Lee, S.J.; Cho, B.J.; Lo, G.Q.; Kwong, D.L. Integration of tensile-strained Ge p-i-n photodetector on advanced CMOS platform. In Proceedings of the 4th IEEE International Conference on Group IV Photonics 2007, Tokyo, Japan, 19-21 September 2007; pp 1-3.

33. Liu, J.; Michel, J.; Giziewicz, W.; Pan, D.; Wada, K.; Cannon, D.; Jongthammanurak, S.; Danielson, D.; Kimerling, L.; Chen, J. High-performance, tensile-strained Ge pin photodetectors on a Si platform. Appl. Phys. Lett. 2005, 87, 103501-103504.

34. Zang, H.; Lee, S.; Loh, W.; Wang, J.; Chua, K.; Yu, M.; Cho, B.; Lo, G.; Kwong, D. Dark-current suppression in metal-germanium-metal photodetectors through dopant-segregation in NiGe-Schottky barrier. IEEE Electr. Dev. Lett. 2008, 29, 161-164.

35. Zang, H.; Lee, S.; Loh, W.; Wang, J.; Yu, M.; Lo, G.; Kwong, D.; Cho, B. Application of dopant segregation to metal-germanium-metal photodetectors and its dark current suppression mechanism. Appl. Phys. Lett. 2008, 92, 051110:1-051110:3.

36. Zang, H.; Lee, S.; Yu, M.; Loh, W.; Wang, J.; Lo, G.; Kwong, D. High-speed metal-germanium-metal configured PIN-like Ge-photodetector under photovoltaic mode and with dopant-segregated Schottky-contact engineering. IEEE Photon. Technol. Lett. 2008, 20, 1965-1967.

37. Ang, K.; Zhu, S.; Wang, J.; Chua, K.; Yu, M.; Lo, G.; Kwong, D. Novel silicon-carbon (Si: C) Schottky barrier enhancement layer for dark-current suppression in Ge-on-SOI MSM photodetectors. IEEE Electr. Dev. Lett. 2008, 29, 704-707.

38. Koester, S.; Schow, C.; Schares, L.; Dehlinger, G.; Schaub, J.; Doany, F.; John, R. Ge-on-SOI-detector/Si-CMOS-amplifier receivers for high-performance optical-communication applications. J. Lightwave Technol. 2007, 25, 46-57.

39. Koester, S.; Schaub, J.; Dehlinger, G.; Chu, J. Germanium-on-SOI infrared detectors for integrated photonic applications. IEEE J. Sel. Top. Quantum Electr. 2007, 12, 1489-1502.

40. Vivien, L.; Rouvière, M.; Fédéli, J.; Marris-Morini, D.; Damlencourt, J.; Mangeney, J.; Crozat, P.; El Melhaoui, L.; Cassan, E.; Le Roux, X. High speed and high responsivity germanium photodetector integrated in a Silicon-On-Insulator microwaveguide. Opt. Express 2007, 15, 9843-9848.

41. Cassan, E.; Marris, D.; Rouviere, M.; Laval, S.; Vivien, L.; Koster, A. Comparison between electrical and optical clock distribution for CMOS integrated circuits. In Proceedings of SPIE 2004, Denver, CO, USA, 1 August 2004; pp. 89-100. 
42. Muoi, T. Receiver design for high-speed optical-fiber systems. J. Lightwave Technol. 1986, 2, 243-267.

43. Kato, K. Ultrawide-band/high-frequency photodetectors. IEEE Trans. Microwave Theory 1999, 47, 1265-1281.

44. Sze, S.; Ng, K. Physics of Semiconductor Devices; Wiley-Blackwell: Boston, MA, USA, 2007.

45. Dosunmu, O.; Cannon, D.; Emsley, M.; Kimerling, L.; Unlu, M. High-speed resonant cavity enhanced Ge photodetectors on reflecting Si substrates for 1,550-nm operation. IEEE Photon. Technol. Lett. 2005, 17, 175-177.

46. Dosunmu, O.; Emsley, M.K.; Cannon, D.D.; Ghyselen, B.; Kimerling, L.C.; Unlu, M.S. Germanium on Double-SOI Photodetectors for 1,550 $\mathrm{nm}$ Operation; Lasers and Electro-Optics Society (LEOS): Piscataway, NJ, USA, 2003; In Proceedings of the 16th Annual Meeting of the IEEE, Tucson, AZ, USA, 27-28 October 2003; Volume 852, pp. 853-854.

47. Yin, T.; Cohen, R.; Morse, M.; Sarid, G.; Chetrit, Y.; Rubin, D.; Paniccia, M. 31 GHz Ge nip waveguide photodetectors on Silicon-on-Insulator substrate. Opt. Express 2007, 15, 13965-13971.

48. Wang, J.; Loh, W.; Chua, K.; Zang, H.; Xiong, Y.; Tan, S.; Yu, M.; Lee, S.; Lo, G.; Kwong, D. Low-voltage high-speed $(18 \mathrm{GHz} / 1 \mathrm{~V})$ evanescent-coupled thin-film-Ge lateral PIN photodetectors integrated on Si waveguide. IEEE Photon. Technol. Lett. 2008, 20, 1485-1487.

49. Chen, L.; Lipson, M. Ultra-low capacitance and high speed germanium photodetectors on silicon. Opt. Express 2009, 17, 7901-7906.

50. Klinger, S.; Berroth, M.; Kaschel, M.; Oehme, M.; Kasper, E. Ge-on-Si p-i-n photodiodes with a 3-dB bandwidth of $49 \mathrm{GHz}$. IEEE Photon. Technol. Lett. 2009, 21, 920-922.

51. Dongwoo, S.; Jiho, J.; Sanghoon, K.; Gyungock, K. High-speed RPCVD Ge waveguide photodetector. In Proceedings of the 6th IEEE International Conference on Group IV Photonics (GFP' 09), San Francisco, CA, USA, 9-11 September 2009; pp. 16-18.

52. Assefa, S.; Fengnian, X.; Vlasov, Y.A. CMOS-integrated low-noise germanium waveguide avalanche photodetector operating at $40 \mathrm{Gbps}$. In Proceedings of Optical Fiber Communication Conference and Exposition and the National Fiber Optic Engineers Conference (OFC/NFOEC 2010), Los Angeles, CA, USA, 21-25 March 2010; pp. 1-3.

53. Kang, Y.; Liu, H.; Morse, M.; Paniccia, M.; Zadka, M.; Litski, S.; Sarid, G.; Pauchard, A.; Kuo, Y.; Chen, H. Monolithic germanium/silicon avalanche photodiodes with $340 \mathrm{GHz}$ gain-bandwidth product. Nat. Photon. 2008, 3, 59-63.

54. Ang, K.; Ng, J.; Lim, A.; Yu, M.; Lo, G.; Kwong, D. Waveguide-integrated Ge/Si avalanche photodetector with $105 \mathrm{GHz}$ gain-bandwidth product. In Proceedings of Optical Fiber Communication Conference and Exposition and the National Fiber Optic Engineers Conference (OFC/NFOEC 2010), Los Angeles, CA, USA, 21-25 March 2010.

55. Assefa, S.; Xia, F.; Vlasov, Y. Reinventing germanium avalanche photodetector for nanophotonic on-chip optical interconnects. Nature 2010, 464, 80-84.

56. Wang, J.; Loh, W.; Zang, H.; Yu, M.; Chua, K.; Loh, T.; Ye, J.; Yang, R.; Wang, X.; Lee, S. Integration of tensile-strained Ge pin photodetector on advanced CMOS platform. In Proceedings of the 4th IEEE International Conference on Group IV Photonics 2007, Tokyo, Japan, 19-21 September 2007; pp. 1-3. 
57. Loh, W.; Wang, J.; Ye, J.; Yang, R.; Nguyen, H.; Chua, K.; Song, J.; Loh, T.; Xiong, Y.; Lee, S. Impact of local strain from selective epitaxial germanium with thin $\mathrm{Si} / \mathrm{SiGe}$ buffer on high-performance pin photodetectors with a low thermal budget. IEEE Electron. Dev. Lett. 2007, 28, 984-986.

58. Loh, T.; Nguyen, H.; Murthy, R.; Yu, M.; Loh, W.; Lo, G.; Balasubramanian, N.; Kwong, D.; Wang, J.; Lee, S. Selective epitaxial germanium on silicon-on-insulator high speed photodetectors using low-temperature ultrathin $\mathrm{Si} 0.8 \mathrm{Ge} 0.2$ buffer. Appl. Phys. Lett. 2007, 91, 073503:1-073503:3.

59. Ang, K.; Yu, M.; Zhu, S.; Chua, K.; Lo, G.; Kwong, D. Novel NiGe MSM photodetector geaturing asymmetrical Schottky barriers using sulfur Co-implantation and segregation. IEEE Electron. Dev. Lett. 2008, 29, 708-710.

60. Masini, G.; Colace, L.; Assanto, G.; Luan, H.; Kimerling, L. High-performance pin Ge on Si photodetectors for the near infrared: from model to demonstration. IEEE Trans. Electron. Dev. 2001, 48, 1092-1096.

61. Rouvière, M.; Vivien, L.; Le Roux, X.; Mangeney, J.; Crozat, P.; Hoarau, C.; Cassan, E.; Pascal, D.; Laval, S.; Fédéli, J. Ultrahigh speed germanium-on-silicon-on-insulator photodetectors for 1.31 and 1.55 m operation. Appl. Phys. Lett. 2005, 87, 231109:1-231109:3.

62. Liu, J.; Cannon, D.; Wada, K.; Ishikawa, Y.; Jongthammanurak, S.; Danielson, D.; Michel, J.; Kimerling, L. Tensile strained Ge pin photodetectors on $\mathrm{Si}$ platform for $\mathrm{C}$ and $\mathrm{L}$ band telecommunications. Appl. Phys. Lett. 2005, 87, 011110:1-011110:3.

63. Jutzi, M.; Berroth, M.; Wöhl, G.; Oehme, M.; Kasper, E. Zero biased Ge-on-Si photodetector on a thin buffer with a bandwidth of $3.2 \mathrm{GHz}$ at 1,300 nm. Mater. Sci. Semicond. Process. 2005, 8, 423-427.

64. Oehme, M.; Werner, J.; Kasper, E.; Jutzi, M.; Berroth, M. High bandwidth Ge pin photodetector integrated on Si. Appl. Phys. Lett. 2006, 89, 071117:1-071117:3.

65. Liu, J.F.; Pan, D.; Jongthammanurak, S.; Ahn, D.; Hong, C.Y.; Beals, M.; Kimerling, L.C.; Michel, J.; Pomerene, A.T.; Hill, C.; Jaso, M.; Tu, K.Y.; Chen, Y.K.; Patel, S.; Rasras, M.; White, A.; Gill, D.M. Waveguide-Integrated Ge p-i-n Photodetectors on SOI Platform. In Proceedings of 3rd IEEE International Conference on Group IV Photonics 2006, Ottawa, Canada, 13-15 September 2006; pp. 173-175.

66. Donghwan, A.; Ching-Yin, H.; Jifeng, L.; Beals, M.; Jian, C.; Kaertner, F.X.; Kimerling, L.C.; Michel, J. Ge photodetectors integrated with waveguides for electronic-photonic integrated circuits on CMOS platform. In Proceedings of Optical Fiber Communication Conference and Exposition and the National Fiber Optic Engineers Conference (OFC/NFOEC 2007), Anaheim, CA, USA, 25-29 March 2007; pp. 1-3.

67. Liu, J.; Ahn, D.; Hong, C.; Pan, D.; Jongthammanurak, S.; Beals, M.; Kimerling, L.; Michel, J.; Pomerene, A.; Carothers, D. Waveguide integrated Ge pin photodetectors on a silicon-on-insulator platform. In Proceedings of 2006 Optics Valley of China International Symposium on Optoelectronics, Wuhan, China, November 2006; pp. 1-4.

68. Wang, J.; Loh, W.; Chua, K.; Zang, H.; Xiong, Y.; Loh, T.; Yu, M.; Lee, S.; Lo, G.; Kwong, D. Evanescent-coupled Ge pin photodetectors on Si-waveguide with SEG-Ge and comparative study of lateral and vertical pin configurations. IEEE Electron. Dev. Lett. 2008, 29, 445-448. 
69. Shiyang, Z.; Kah-Wee, A.; Rustagi, S.C.; Wang, J.; Xiong, Y.Z.; Lo, G.Q.; Kwong, D.L. Waveguided $\mathrm{Ge} / \mathrm{Si}$ avalanche photodiode with separate vertical SEG-Ge absorption, lateral Si charge, and multiplication configuration. IEEE Electron. Dev. Lett. 2009, 30, 934-936.

70. Tao, Y.; Cohen, R.; Morse, M.M.; Sarid, G.; Chetrit, Y.; Rubin, D.; Paniccia, M.J. 40 Gb/s Ge-on-SOI waveguide photodetectors by selective Ge growth. In Proceedings of Optical Fiber Communication Conference and Exposition and the National Fiber Optic Engineers Conference (OFC/NFOEC 2008), San Diego, CA, USA, 24-28 Feburary 2008; pp. 1-3.

71. Assefa, S.; Xia, F.; Bedell, S.; Zhang, Y.; Topuria, T.; Rice, P.; Vlasov, Y. CMOS-integrated high-speed MSM germanium waveguide photodetector. Opt. Express 2010, 18, 4986-4999.

72. Dazeng, F.; Shirong, L.; Po, D.; Ning-Ning, F.; Dawei, Z.; Hong, L.; Shafiiha, R.; Guoliang, L.; Cunningham, J.; Raj, K.; Krishnamoorthy, A.V.; Asghari, M. Horizontal p-i-n high-speed Ge waveguide detector on large cross-section SOI waveguide. In Proceedings of Optical Fiber Communication Conference and Exposition and the National Fiber Optic Engineers Conference (OFC/NFOEC 2010), Los Angeles, CA, USA, 21-25 March 2010; pp. 1-3.

73. Suh, D.; Joo, J.; Kim, S.; Kim, G. High-speed RPCVD Ge waveguide photodetector. In Proceedings of the 6th IEEE International Conference on Group IV Photonics (GFP' 09), San Francisco, CA, USA, 9-11 September 2009; pp. 16-18.

74. Pavesi, L.; Lockwood, D. Silicon Photonics, Topics in Applied Physics; Springer-Verlag: Berlin, Germany, 2004; Volume 94.

75. Ang, K.; Liow, T.; Yu, M.; Fang, Q.; Song, J.; Lo, G.; Kwong, D. Low thermal budget monolithic integration of evanescent-coupled Ge-on-SOI photodetector on Si CMOS platform. IEEE J. Sel. Top. Quantum Electron. 2010, 16, 106-113.

76. Schuller, J.; Barnard, E.; Cai, W.; Jun, Y.; White, J.; Brongersma, M. Plasmonics for extreme light concentration and manipulation. Nat. Mater. 2010, 9, 193-204.

77. Ishi, T.; Fujikata, J.; Makita, K.; Baba, T.; Ohashi, K. Si nano-photodiode with a surface plasmon antenna. Jpn. J. Appl. Phys. 2005, 44, L364-L366.

78. Ren, F.; Ang, K.; Lo, G.; Kwong, D. Nanometer germanium photodetector with aluminum surface plasmon antenna for enhanced photo-response. Proc. SPIE 2010, doi:10.1117/12.854179.

79. Ang, K.; Liow, T.; Fang, Q.; Yu, M.; Ren, F.; Zhu, S.; Zhang, J.; Ng, J.; Song, J.; Xiong, Y.; Lo, G.Q.; Kwong, D.-L. Silicon photonics technologies for monolithic electronic-photonic integrated circuit (EPIC) applications: Current progress and future outlook. In Proceedings of 2009 IEEE International Electron Devices Meeting (IEDM), Baltimore, MD, USA; 7-9 December 2009; pp. 1-4.

(C) 2011 by the authors; licensee MDPI, Basel, Switzerland. This article is an open access article distributed under the terms and conditions of the Creative Commons Attribution license (http://creativecommons.org/licenses/by/3.0/). 